DOI: http://dx.doi.org/10.12957/demetra.2015.13479

\title{
Aleitamento materno exclusivo e alimentação complementar entre menores de um ano em Ribeirão das Neves-MG
}

\section{Exclusive breastfeeding and supplementary feeding among children under one year old in Ribeirão das Neves, Minas Gerais state, Brazil}

Meire Rafaela dos Santos Gonçalves' Matilde Meire Miranda Cadete²

' Centro Universitário UNA, Curso de Mestrado Profissional em Gestão Social, Educação e Desenvolvimento Local. Belo Horizonte-MG, Brasil.

Correspondência / Corrrespondence Meire Rafaela dos Santos Gonçalves E-mail:meirerafaela@yahoo.com.br

\section{Resumo}

Objetivo: A pesquisa buscou identificar o índice de aleitamento materno exclusivo (AME) e alimentação complementar adotada em menores de um ano no município de Ribeirão das NevesMG. Material e métodos: Estudo realizado em dez unidades básicas de saúde do município, cuja coleta de dados se fez por meio de questionário composto de 55 questões para responsáveis e/ou acompanhantes das crianças menores de um ano, que compareceram à sala de vacina no período do estudo. Foram distribuídos 353 questionários nas unidades de saúde. A análise desses dados se fez por meio das associações das variáveis com AME baseadas nos testes qui-quadrado de Pearson assintótico e exato, e testes qui-quadrado de Pearson exato com as variáveis do estudo. Resultados: O índice de AME no município foi de 30\%, exigindo o desenvolvimento de atividades, programas ou serviço de promoção, apoio e aconselhamento em AM. As variáveis que tiveram correlação com o baixo índice de AM no município foram o uso de mamadeira e a idade da criança. Identificaram-se o início precoce da alimentação complementar e a utilização de alimentos não recomendados para a idade da criança, práticas que podem estar ligadas a fatores culturais. Conclusão: A introdução de precoce de alimentos não recomendados para a idade favorece o desmame precoce, além do estabelecimento de um hábito alimentar não saudável na vida adulta. Os dados sugerem que a equipe multidisciplinar deve estar preparada para atender às necessidades individuais da mãe e da criança para acompanhamento e avaliação do AME ou da alimentação complementar.

Palavras-chave: Suplementação Alimentar. Comportamento Alimentar. Aleitamento Materno. Nutrição do Lactente. 


\section{Abstract}

Objective: The study aimed to identify the rate of exclusive breastfeeding and supplementary feeding adopted among children aged under a year in the city of Ribeirão das Neves-MG, Brazil. Material and methods: Research conducted in 10 municipal basic health units, with data collected through a questionnaire with 55 questions for those responsible and/or chaperones of children under one year, who attended the vaccine room in the study period. A total of 353 questionnaires were distributed among the health units. Data analysis comprised association of variables with exclusive breastfeeding based on chi-square tests of Pearson exact and asymptotic and chi-square tests of Pearson accurate with the study variables. Results: The index of exclusive breastfeeding in the city was $30 \%$, requiring development of activities, programs or service to promote, support and advice in breastfeeding. The variables that have correlation with the low content of breastfeeding in the municipality were use of bottle and age of the child. We identified early supplementary feeding and use of foods not recommended for the age of the child, practice that can be linked to cultural factors. Conclusion: Early introduction of foods not recommended for age favors early weaning in addition to promoting the establishment of a healthy food habit in adulthood. Data suggest that the multidisciplinary team should be prepared to meet the individual needs of mother and child for monitoring and evaluating breastfeeding or supplementary feed.

Key words: Food Supplementation. Feeding Behavior. Breastfeeding. Infant Nutrition.

\section{Introdução}

O aleitamento materno (AM) é determinado social e culturalmente; tem a indicação de ser realizado de forma exclusiva até os seis meses de idade da criança e complementado até os dois anos de idade. Ele aumenta o vínculo entre mãe e filho e previne contra doenças e agravos à saúde., ${ }^{1,2}$

O AM sofreu grande influência durante o processo de industrialização do Brasil, onde os trabalhadores eram sobrecarregados tanto de quantidade de trabalho, quanto da carga horária destinada ao trabalho. Kemp considera que "[o] objectivo era garantir que 
os trabalhadores prestassem a máxima atenção à tarefa que lhes competia. As operações que realizavam e o ritmo do seu trabalho submetiam-se ao próprio ritmo da máquina" (p. 33). O autor ainda reforça a "utilização de mão-de-obra feminina e infantil barata".

Após a revolução industrial, a mulher tornou-se trabalhadora formal, ficando os filhos aos cuidados de outrem, interferindo negativamente na manutenção do AM. Pode-se ponderar que, após a Revolução Industrial, a mulher passou a ocupar espaços anteriormente destinados somente aos homens, e essa inserção no mercado de trabalho produziu consequências sociais também no núcleo familiar. Houve, com isso, por um lado, sobrecarga da mulher com jornada dupla ou tripla de trabalho e, por outro, mudanças na estrutura emocional da família. Os filhos são precocemente encaminhados para os cuidados de outrem, que muitas das vezes não possuem vínculo afetivo com a criança e esta, muito cedo, deve se acostumar com a ausência materna.

A utilização do leite em pó e outros alimentos industrializados para as crianças tornouse mais comum após o período de industrialização. Desde então e até os dias atuais, o leite em pó mantém sua imagem associada a um produto que promove boa nutrição, com repercussões negativas sobre o aleitamento materno. ${ }^{4}$

De acordo com Frota, ${ }^{2}$ dificuldades da mãe na interpretação do choro da criança, problemas com as mamas, recusa do peito pela criança e quantidade insuficiente de leite materno também podem interferir na manutenção da amamentação. Na concepção de Venâncio \& Monteiro, ${ }^{5}$

deve-se considerar inicialmente que a alimentação artificial tornou-se não somente uma opção para as mulheres, mas uma prática amplamente aceita. Neste contexto, a prática da amamentação precisa ser socialmente reconstruída (p. 47).

A despeito da existência de políticas públicas que estimulam o aleitamento materno, observa-se com frequência, na prática assistencial, que gestantes chegam aos hospitais ainda carentes de orientação sobre o AM e, ao receberem alta hospitalar, não contam com serviço de referência para o acompanhamento, orientação e suporte pós-natal do AM. ${ }^{1}$ Tais condições terminam por favorecer o desmame precoce e a introdução de outros alimentos não recomendados para a idade do lactente (como amido de milho, farinha de arroz, café, dentre outros). Pode-se, a partir desse cenário, inferir que as unidades básicas de saúde (UBSs) do município de Ribeirão das Neves não estão desenvolvendo as ações de promoção, proteção e apoio ao AM, conforme estabelecido pela Política Nacional de Promoção, Proteção e Apoio ao Aleitamento Materno.

Em todos os níveis de atenção à saúde, destaca-se a necessidade de respeitar as especificidades contidas nos cuidados integrais, quer de atenção, gestão e educação às 
gestantes/nutrizes. Um dos instrumentos para acompanhar a atenção à saúde no Brasil é o Sistema de Informação da Atenção Básica (SIAB), que também possibilita aproximações na prevalência de aleitamento materno na população. ${ }^{6}$ Deve-se, entretanto, considerar limitações importantes na qualidade das informações aí disponibilizadas. Por exemplo, de acordo com os dados do SIAB, no ano de 2010, o município de Ribeirão das Neves, no estado de Minas Gerais, apresentou taxa de AME em menores de quatro meses acima de $70 \%$, chegando a atingir índices de 100\%. Um dado questionável, se comparado à prática clínica percebida no município. ${ }^{6}$

Justifica-se, assim, a realização desta investigação acerca da prevalência de aleitamento materno nesse município, o que poderá contribuir para nortear as ações de promoção de saúde em aleitamento materno aí propostas e materializadas.

\section{Material E Métodos}

Um questionário composto de 55 questões foi aplicado aos responsáveis e/ou acompanhantes das crianças menores de um ano que estavam presentes na unidade de saúde. Registra-se que, anteriormente, foram selecionadas dez unidades de saúde, entre as cinco regiões sanitárias do município. A escolha das unidades realizou-se de forma aleatória, após consentimento da Coordenação da Atenção Primária, bem como da Coordenação de Imunização do município. Antes da coleta, realizou-se contato com o(a) enfermeiro(a) da unidade, que foi assim comunicado(a) sobre a pesquisa.

O instrumento de análise foi validado pela Pesquisa Nacional sobre Práticas Alimentares no Primeiro Ano de Vida, que analisa a alimentação da criança nas últimas 24 horas. $^{7}$ A utilização desse instrumento visa identificar o do índice de AM no município e obter dados fidedignos.

A coleta foi realizada durante a campanha de vacinação contra a poliomielite, em 16 de junho de 2012, sendo distribuídos os 353 questionários entre as dez unidades de saúde. Entretanto, não foi possível preencher todos os questionários no dia da campanha de vacinação. Foi necessário retornar a algumas unidades de saúde para aplicar o questionário, e incluir o Centro Viva Vida de Referência Secundária (CVVRS) na coleta de dados.

O CVVRS é um serviço de Atenção Secundária à Saúde, atendendo a casos de prematuridade, baixo-peso ao nascer, desnutrição, triagem neonatal positiva e outros agravos relacionados ao nascimento ou à primeira infância de relevância clínica. A 
inclusão do CVVRS fez-se imperiosa, por se tratar de uma unidade que atende a todo o município. Na sua dinâmica de trabalho, instituiu que o atendimento se faz por meio de consultas agendadas e a um público específico (considerando os critérios clínicos para o encaminhamento), tornando-se relevante identificar os índices de AME e a prática da alimentação complementar nesse grupo de risco.

A reaplicação do questionário incidiu até o dia 21 de agosto de 2012, quando se atingiu a coleta do número total de questionários. Todos os entrevistados foram informados da natureza e teor da pesquisa, autorizando verbalmente a aplicação do questionário.

Foram definidos 353 questionários, com erro amostral de 5\%, com N=4.307 de crianças menores de um ano no município (de acordo com informação oferecida pela coordenadora do Setor de Imunização da Secretaria Municipal de Saúde, a partir dos dados do SIAB) e intervalo de confiança de $50 \%$, a partir do cálculo: ${ }^{8}$

$$
\mathrm{n}=\frac{\mathrm{z}^{2} \cdot \sigma^{2} \cdot N}{\mathrm{~d}^{2}(N-1)+Z^{2} \cdot \sigma^{2}}
$$

Onde: $\mathrm{Z}=$ abcissa normal padrão

$\sigma^{2} \sigma^{2}=$ variância populacional

$\mathrm{N}=$ tamanho da população

$\mathrm{d}=$ erro amostral

Os dados foram analisados no SPSS versão 15.0. A caracterização dos dados foi realizada por meio de frequências absolutas e porcentagens e de gráficos de barras. As análises de associações foram realizadas através dos testes qui-quadrado de Pearson assintótico e exato. Para localizar onde se encontra a associação, foi calculado o resíduo ajustado padronizado: se o valor do resíduo fosse menor ou igual a -1,96, e valor do resíduo maior ou igual a $+1,96$.

As análises das associações das variáveis com AME foram baseadas nos testes quiquadrado de Pearson assintótico e exato, e testes qui-quadrado de Pearson exato com as variáveis do estudo. 


\section{Resultado e discussão}

o município de Ribeirão das Neves é dividido em cinco regiões sanitárias, com vistas a descentralização e regionalização das ações de saúde. As regiões sanitárias foram dividas geograficamente, buscando atender às necessidades regionais de cada grupo da população. Assim, os dados foram colhidos em todas as regiões sanitárias e no CVVRS, para identificar se havia diferenças acentuadas da prática alimentar e manutenção do AME entre as regiões do município.

A coleta de dados nas regiões sanitárias ficou caracterizada da seguinte forma: 14,2\% pertencem ao CVVRS; 11,3\% à região I; 10,5\% à região II; 25,8\% à região III; 22,7\% à região IV; e 15,6\% à região V. Nota-se uma coleta maior nas regiões III e IV, dado que pode ser justificado pela região geográfica, que possui grande número de moradores e também pelo maior número de crianças presentes na campanha de vacinação.

Entrevistaram-se as mães ou responsáveis pela criança, dos quais $87,8 \%$ eram mães e 12,2\%, responsáveis pelas crianças. Quanto à idade das crianças, $12,5 \%$ estavam entre 1-29 dias; $40,2 \%$, de 1 a 3 meses e 29 dias; $17,3 \%$ entre 4 e 5 meses e 29 dias; e $30 \%$ estavam entre 6 e 12 meses completos.

Em relação ao sexo, há pequena predominância do sexo masculino, representado por $50,7 \%$ das crianças analisadas. E quanto ao hábito alimentar, $74,2 \%$ tomaram leite do peito nas últimas 24 horas, e destes que aleitaram, 48,2\% amamentaram no peito oito vezes ou mais. Do universo analisado, $62,3 \%$ das crianças tomaram água; $44,2 \%$, chá; e 55,5\% ingeriram outro leite nas últimas 24 horas que antecederam a entrevista.

Mais de $50 \%$ das crianças que participaram da pesquisa encontravam-se na faixa etária de até cinco meses e 29 dias. O ideal seria que esta mesma proporção estivesse em AME. Entretanto, houve alto índice de ingestão de água e outro leite. Dado semelhante foi encontrado por Arantes et al., ${ }^{9}$ ao identificarem que a introdução de outro leite ocorreu precocemente a partir dos três meses de idade, apresentando uma curva crescente até os cinco meses; perceberam também uma correlação estatística entre o consumo de água, chá e sucos e a suspensão do AME.

Para identificar o índice de AME, as variáveis da alimentação da criança nas últimas 24 horas foram cruzadas com a variável "tomou leite do peito". A criança que aleita exclusivamente deve receber a resposta "não" para todas as variáveis da alimentação complementar. Os resultados foram descritos na tabela 1. 
Tabela 1. Alimentação da criança nas últimas 24 horas, Ribeirão das Neves-MG, 2012.

\begin{tabular}{|c|c|}
\hline Variáveis & $\mathrm{n}(\%)$ \\
\hline \multicolumn{2}{|l|}{ Tomou leite do peito } \\
\hline Sim & $262(74,2)$ \\
\hline Não & $91(25,8)$ \\
\hline \multicolumn{2}{|c|}{ Quantas vezes tomou leite do peito } \\
\hline 1 a 3 vezes & $21(5,9)$ \\
\hline 4 a 7 vezes & $44(12,5)$ \\
\hline 8 vezes ou mais & $170(48,2)$ \\
\hline Não sabe & $28(7,9)$ \\
\hline Sem informação & $90(25,5)$ \\
\hline \multicolumn{2}{|l|}{ Tomou água } \\
\hline Sim & $220(62,3)$ \\
\hline Não & $133(37,7)$ \\
\hline Não sabe & $0(0,0)$ \\
\hline \multicolumn{2}{|l|}{ Tomou chá } \\
\hline Sim & $156(44,2)$ \\
\hline Não & $196(55,5)$ \\
\hline Não sabe & $1(0,3)$ \\
\hline \multicolumn{2}{|l|}{ Tomou outro leite } \\
\hline Sim & $196(55,5)$ \\
\hline Não & $154(43,6)$ \\
\hline Não sabe & $3(0,8)$ \\
\hline \multicolumn{2}{|c|}{ A criança recebeu outro leite } \\
\hline Só durante o dia & $41(11,6)$ \\
\hline Só durante a noite & $26(7,4)$ \\
\hline De dia e de noite & $131(37,1)$ \\
\hline Não sabe & $2(0,6)$ \\
\hline Sem informação & $153(43,3)$ \\
\hline \multicolumn{2}{|c|}{ Tomou mingau doce ou salgado } \\
\hline Sim & $98(27,7)$ \\
\hline Não & $254(72,0)$ \\
\hline Não sabe & $1(0,3)$ \\
\hline
\end{tabular}




\begin{tabular}{cc}
\hline Variáveis & $\mathrm{n}(\%)$ \\
\hline Sim & $169(47,9)$ \\
Não & $184(52,1)$ \\
Não sabe & $0(0,0)$ \\
\hline Comeu papa salgada & \\
Sim & $161(45,6)$ \\
Não & $191(54,1)$ \\
Não sabe & $1(0,3)$ \\
Tomou suco de fruta & $178(50,4)$ \\
Sim & $174(49,3)$ \\
Não & $1(0,3)$ \\
Não sabe & $37(10,5)$ \\
Tomou suco industrializado & $315(89,2)$ \\
Sim & $1(0,3)$ \\
Não &
\end{tabular}

A prevalência de AME no município de Ribeirão das Neves é de 30\%, muito aquém do preconizado pela OMS e muito abaixo do índice nacional, que é de $41 \% .^{7}$

Identificou-se prevalência de AME em crianças com idade até três meses e 29 dias, corroborando o estudo de Filamingo, Lisboa \& Basso, ${ }^{10}$ que encontrou prevalência de AME de 74,4\% em crianças de até quatro meses de idade e de 15,4\% em crianças com seis meses de idade.

Resultado semelhante também foi localizado em outo estudo, em que 63,6\% das crianças analisadas foram desmamadas antes de completar seis meses de idade, sendo esse desmame precoce avaliado como consequência da introdução precoce do leite não materno na dieta alimentar da criança. ${ }^{11}$

O AM foi mantido em 74,2\% na dieta alimentar das crianças analisadas, seja de forma complementada (quando a criança recebe outros alimentos sólidos ou semissólidos), predominante (quando a criança recebe bebidas à base de água), mista (quando recebe outro tipo de leite) ou somente aleitamento materno (quando a criança recebe o leite materno independentemente de receber ou não outros alimentos). As definições do 
tipo de aleitamento materno adotadas seguem o padrão da OMS, que também define que o AM deve ser mantido até os dois anos ou mais da criança. ${ }^{1}$ De certa forma, a população estudada segue as orientações do Ministério da Saúde, ao adotar o AM, independentemente da classificação do mesmo.

Das crianças que tomaram outro leite, ou seja, 55,5\% dos entrevistados, 37,1\% o receberam de dia e de noite.

$\mathrm{Na}$ alimentação das últimas 24 horas, as mães ou responsáveis indicaram que $27,7 \%$ tomaram mingau salgado; $47,9 \%$ comeram fruta; $45,6 \%$ comeram papa salgada; $50,4 \%$ tomaram suco de fruta; $10,5 \%$ receberam suco industrializado; $10,5 \%$ tomaram refrigerante; $9,3 \%$ tomaram café, $39,1 \%$ receberam alimento adoçado com açúcar; 35, 1\% comeram bolacha ou salgadinho; $21,5 \%$ tomaram ou comeram outros alimentos. No que diz respeito à mamadeira, $65,2 \%$ usaram-na ou chuquinha e $51 \%$ fizeram uso da chupeta.

A introdução da alimentação complementar deve basear-se em uma alimentação saudável, rica em nutrientes, frutas e verduras. O consumo de produtos industrializados, ricos em sal, açúcar ou gordura deve ser evitado sobretudo no primeiro ano de vida da criança. ${ }^{12}$

Como se pode notar, a introdução de alimentos ricos em açúcar, sal, gordura é expressiva na população estudada. O consumo de frutas não alcança $50 \%$ da população, levando a refletir se as mães estão sendo orientadas corretamente sobre a introdução da alimentação complementar.

Em estudo sobre a alimentação complementar em lactentes, os autores, ao compararem a alimentação em menores e maiores de seis meses, detectaram, respectivamente, que o consumo de refrigerante foi de 0 e 9\%; o consumo de bolacha foi de 38,5 e 79,3\%; o consumo de suco artificial, 1,5 e 20,7\%; e o consumo de frutas foi de 84,6 e $97,3 \% .{ }^{13}$ Os dados são semelhantes aos encontrados nesta pesquisa, sendo relevante ressaltar que o consumo de frutas foi muito superior ao identificado na população de Ribeirão das Neves.

A correta diluição da fórmula infantil oferecida à criança é um dado importante a ser inquerido às mulheres que oferecem esse tipo de complemento à dieta do filho. Um estudo identificou que a diluição adequada da fórmula infantil foi de 23,8\% para menores de seis meses e de 34,7 para maiores de seis meses. Considerando a diluição do leite de vaca, o mesmo estudo indicou que 3,9\% das crianças menores de seis meses recebiam uma diluição correta deste leite, contra $15,3 \%$ para as crianças maiores de seis meses. ${ }^{13}$ A população estudada possui um índice de $37,1 \%$ de crianças que recebem outro leite, o que convoca para a importância de se avaliar a diluição, preparo e administração deste, a fim de garantir uma nutrição adequada dos lactentes. 
Tabela 2. Resultados da análise de associação entre idade da criança e o hábito alimentar, Ribeirão das Neves-MG, 2012

\begin{tabular}{|c|c|c|c|c|c|c|}
\hline \multirow[t]{2}{*}{ Variáveis } & \multicolumn{4}{|c|}{ Idade da criança } & \multirow[t]{2}{*}{ Total } & \multirow[t]{2}{*}{ Valor-p } \\
\hline & 1 a 29 dias & $\begin{array}{c}\text { De 1mês a } 3 \\
\text { meses e } 29 \text { dias }\end{array}$ & $\begin{array}{c}\text { De } 4 \text { a } 5 \text { meses } \\
\text { e } 29 \text { dias }\end{array}$ & $\begin{array}{c}\text { De } 6 \text { a } 12 \\
\text { meses }\end{array}$ & & \\
\hline \multicolumn{7}{|l|}{ Tomou água } \\
\hline Sim & $5(11,4)^{*}$ & $64(45,1) *$ & $53(86,9) * *$ & $98(92,5) * *$ & 220 & $<0,00011$ \\
\hline Não & $39(88,6) * *$ & $78(54,9) * *$ & $8(13,1) *$ & $8(7,5) *$ & 133 & \\
\hline \multicolumn{7}{|l|}{ Tomou chá } \\
\hline Sim & $12(27,3)^{*}$ & $64(45,1)$ & $28(45,9)$ & $52(49,1)$ & 230 & \\
\hline Não & $32(72,7) * *$ & $77(54,2)$ & $33(54,1)$ & $54(50,9)$ & 123 & 0,2582 \\
\hline Não sabe & $0(0,0)$ & $1(0,7)$ & $0(0,0)$ & $0(0,0)$ & & \\
\hline \multicolumn{7}{|l|}{ Tomou outro leite } \\
\hline Sim & $13(29,5)^{*}$ & $64(45,1) *$ & $41(67,2) * *$ & $78(73,6) * *$ & 196 & 0,9022 \\
\hline Não & $31(70,5) * *$ & $76(53,5) * *$ & $20(32,8)$ & $27(25,5) *$ & 154 & \\
\hline Não sabe & $0(0,0)$ & $2(1,4)$ & $0(0,0)$ & $1(0,0)$ & 3 & \\
\hline \multicolumn{7}{|l|}{$\begin{array}{c}\text { A criança recebeu } \\
\text { outro leite }\end{array}$} \\
\hline Só durante o dia & $3(23,1)$ & $19(28,8) * *$ & $11(26,8)$ & $8(10,0) *$ & 41 & $<0,00012$ \\
\hline Só durante a noite & $5(38,5) * *$ & $13(19,7) * *$ & $3(7,3)$ & $5(6,3) *$ & 26 & \\
\hline De dia e de noite & $5(38,5) *$ & $34(51,5) *$ & $27(65,9)$ & $65(81,3) * *$ & 131 & \\
\hline Não sabe & $0(0,0)$ & $0(0,0)$ & $0(0,0)$ & $2(2,4)$ & 2 & \\
\hline Sem informação & $0(0,0)$ & $0(0,0)$ & $0(0,0)$ & $0(0,0)$ & 0 & \\
\hline \multicolumn{7}{|l|}{$\begin{array}{l}\text { Tomou mingau } \\
\text { doce ou salgado }\end{array}$} \\
\hline Sim & $1(2,3) *$ & $22(15,5) *$ & $23(37,7)$ & $52(49,1) * *$ & 98 & $<0,00012$ \\
\hline Não & $43(97,7) * *$ & $120(84,5) * *$ & $38(62,3)$ & $53(50,0) *$ & 254 & \\
\hline Não sabe & $0(0,0)$ & $0(0,0)$ & $0(0,0)$ & $1(0,0)$ & 1 & \\
\hline \multicolumn{7}{|l|}{$\begin{array}{l}\text { Comeu fruta } \\
\text { em pedaço ou } \\
\text { amassada }\end{array}$} \\
\hline Sim & $1(2,3)$ * & $25(17,6)$ * & $43(70,5) * *$ & $100(94,3) * *$ & 169 & $<0,00011$ \\
\hline Não & $43(97,7) * *$ & $117(82,4) * *$ & $18(29,5) *$ & $6(5,7) *$ & 184 & \\
\hline Não sabe & $0(0,0)$ & $0(0,0)$ & $0(0,0)$ & $0(0,0)$ & 0 & \\
\hline
\end{tabular}




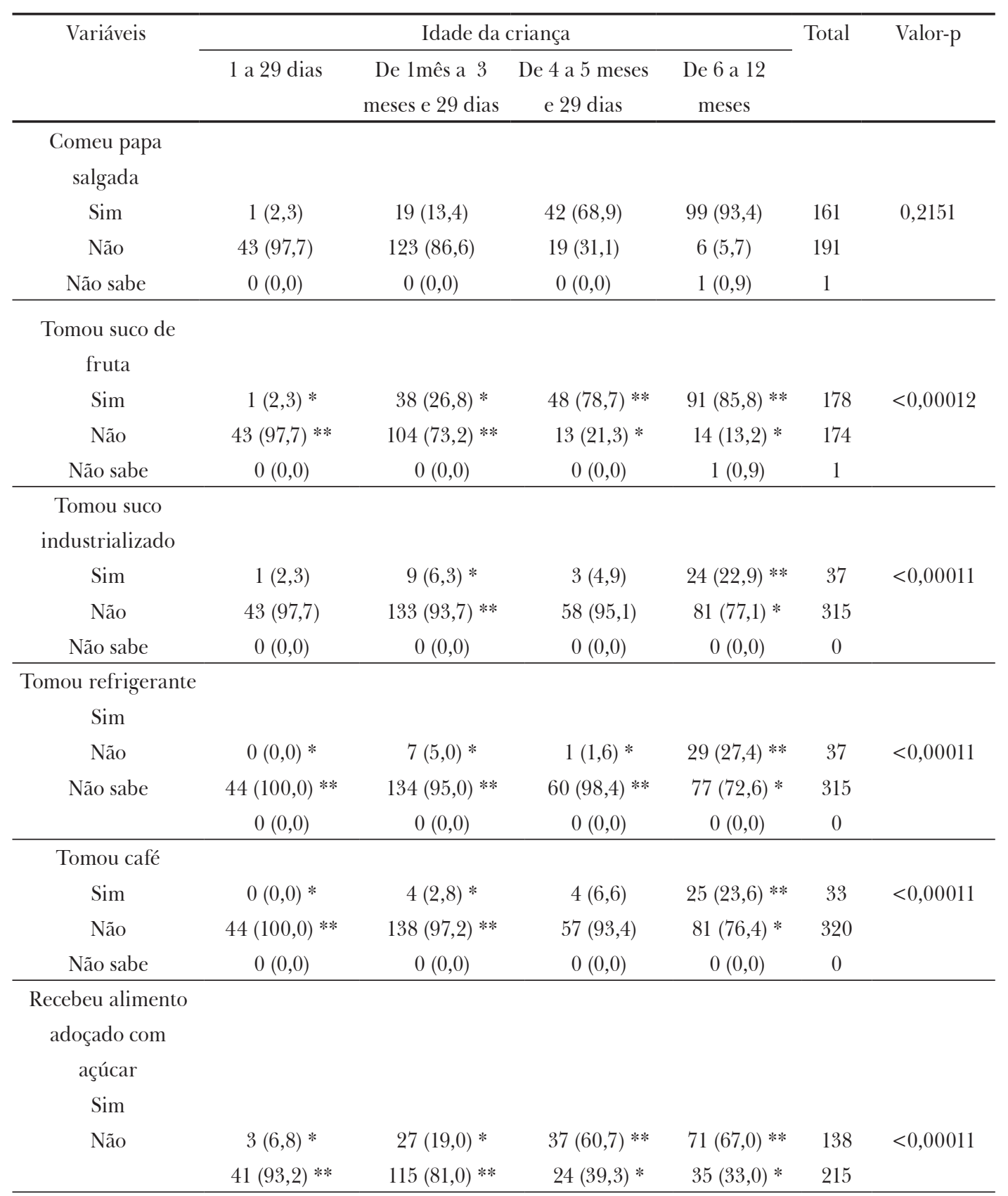




\begin{tabular}{|c|c|c|c|c|c|c|}
\hline \multirow[t]{2}{*}{ Variáveis } & \multicolumn{4}{|c|}{ Idade da criança } & \multirow[t]{2}{*}{ Total } & \multirow[t]{2}{*}{ Valor-p } \\
\hline & 1 a 29 dias & $\begin{array}{c}\text { De } 1 \text { mês a } 3 \\
\text { meses e } 29 \\
\text { dias }\end{array}$ & $\begin{array}{c}\text { De } 4 \text { a } 5 \\
\text { meses e } 29 \\
\text { dias }\end{array}$ & $\begin{array}{c}\text { De } 6 \text { a } 12 \\
\text { meses }\end{array}$ & & \\
\hline
\end{tabular}

Comeu bolacha

ou salgadinho

Sim

$1(2,3) * \quad 15(10,6) *$

$25(41,0)$

$83(78,3) * *$

$124<0,00011$

Não

$43(97,7) * * 127(89,4) * *$

$36(59,0)$

$22(20,8) *$

228

Não sabe

$0(0,0)$

$0(0,0)$

$0(0,0)$

$1(0,9)$

1

Tomou ou

comeu outros

alimentos

$\begin{array}{lcccccc}\text { Sim } & 0(0,0) * & 9(6,3) * & 17(27,9) & 50(47,2) * * & 76 & <0,00011 \\ \text { Não } & 44(100,0) & 133(93,7) * * & 44(72,1) & 55(51,9) * & 276 & \\ \text { Não sabe } & * * & 0(0,0) & 0(0,0) & 1(0,0) & 1 & \\ & 0(0,0) & & & & & \end{array}$

${ }^{1}$ Teste qui-quadrado de Pearson assintótico. ${ }^{2}$ Teste qui-quadrado de Pearson exato. *Resíduo ajustado <-1,96.

**Resíduo ajustado $>1,96$.

Na tabela 2, são descritas a relação entre a variável “idade da criança” e as variáveis do hábito alimentar. As variáveis da alimentação complementar que tiveram associação estatística, de acordo com os valores-p $\leq 0,05$ nos testes qui-quadrado de Pearson assintótico e exato, com a idade da criança, foram: tomar água, receber outro leite, tomar mingau doce ou salgado, comer frutas em pedaço ou amassada, tomar suco de fruta ou suco industrializado, ingerir refrigerante e ou café, receber alimento adoçado com açúcar, comer bolacha ou salgadinho e tomar ou comer outros alimentos.

Segundo a análise de resíduos ajustados, conclui-se que as crianças que mais tomaram água, que mais comeram fruta, que mais tomaram suco de fruta e receberam alimentação adocicada com açúcar estavam com idade entre 4-5 meses e 29 dias e de 6-12 meses. Já as crianças que tomaram mingau doce ou salgado, tomaram sucos industrializados e café, comeram bolacha ou salgadinho e tomaram ou comeram outros alimentos estavam na faixa etária de 6-12 meses.

As crianças menores de um mês de idade que tomaram outro leite o fizeram somente durante a noite; as crianças com idade entre 1-3 meses e 29 dias só ingeriram outro leite durante o dia ou só durante a noite e aquelas entre 6-12 meses, durante dia e noite. 
Ao iniciar precocemente a alimentação complementar, aumenta-se o risco de a criança desenvolver algumas doenças correlacionadas ao desmame precoce. Pode-se citar que o desmame precoce aumenta o risco de desenvolver pneumonia; aumenta o risco do óbito em 2,2 vezes por doenças infecciosas e 3,9\% por diarreia ou doenças respiratórias. ${ }^{14}$

Como já discutido anteriormente, o leite humano é capaz de suprir todas as necessidades nutricionais do bebê até o sexto mês de vida. Quando ocorre a introdução precoce de outros alimentos, sobrevém uma deficiência nutricional na dieta da criança, pois nenhum outro alimento contém as propriedades nutritivas do leite humano, o que pode levar à desnutrição infantil por práticas inadequadas de desmame e introdução de alimentos. ${ }^{14,15}$

O leite de vaca é responsável por $20 \%$ das alergias alimentares e sua introdução na dieta alimentar da criança deve ser postergado o maior tempo possível, sendo sugerida sua introdução a partir de nove meses de idade. ${ }^{13-15}$ Esse leite é rico em proteínas, que são totalmente absorvidas pela mucosa intestinal da criança e, por conseguinte, há uma resposta imunológica a esta proteína íntegra, levando à alergia alimentar. Este leite é também fator de risco independente para o desenvolvimento de anemia carencial ferropriva, sendo responsável por uma queda de $0,2 \mathrm{~g} / \mathrm{dl}$ dos níveis de hemoglobina por mês na criança. ${ }^{13-15} \mathrm{O}$ leite de vaca é também correlacionado com o aparecimento de diarreias, devido à contaminação do leite durante o preparo, doenças atópicas, diabetes mellitus tipo I e asma. Destaca-se, ainda, que a criança que recebe o leite de vaca antes de quatro meses de idade tem risco três vezes maior de desenvolver asma até os quatro anos de idade do que aquelas que não recebem leite de vaca. ${ }^{15,16}$

O consumo precoce de café e chá também está relacionado com a baixa absorção de ferro, sendo desaconselhado seu consumo até que a criança complete um ano de idade. ${ }^{15,16}$ A papa de fruta iniciada antes dos seis meses de idade possui dois fatores relevantes a serem observados: é uma fonte de contaminação quando não higienizada corretamente, podendo levar a diarreias; e devido a imaturidade neurológica da criança até os seis meses, a mesma não consegue deglutir corretamente os alimentos que não são líquidos, podendo levar à aspiração ou engasgo. ${ }^{9,14}$

Em relação ao uso precoce de água e suco de fruta, esses ingredientes se mostraram menos agressivos à criança quando comparados aos outros alimentos. Na vigência da necessidade da introdução precoce de alimentos à dieta da criança ou se a mãe já iniciou a alimentação complementar, os profissionais de saúde devem estar preparados para a oferta de apoio e orientação adequados às necessidades singulares da mãe e da criança. ${ }^{14}$

O consumo de alimentos não nutritivos, como refrigerante, açúcar, bolachas, salgadinhos, doces, frituras e alimentos industrializados, deve ser desestimulado, pois 
eles têm relação com o surgimento de anemia ferropriva, obesidade infantil e alergias alimentares. O sal iodado tem importância significativa na dieta da criança, pois garante a ingestão do iodo; no entanto, seu consumo deve ser moderado. ${ }^{12}$

As situações de insegurança alimentar devem ser avaliadas e acompanhadas prioritariamente. Em outra pesquisa realizada, os autores identificaram que o AM é maior nas crianças que se encontram em situação de insegurança alimentar. ${ }^{17}$ Como já descrito, AM não substitui a alimentação complementar após seis meses de vida da criança, mas nessa situação específica, sua manutenção prolongada, somada à alimentação complementar, favorece a saúde da mesma.

\section{Conclusão}

Nota-se que a prática alimentar em menores de um ano de idade, na presente pesquisa, não está adequada às orientações do Ministério da Saúde. $\mathrm{O}$ alto consumo de alimentos não recomendados para a idade pode contribuir para formação de hábitos alimentares não saudáveis na vida adulta.

O índice de AME do município encontra-se muito inferior ao recomendado. Este cenário faz-nos refletir sobre o envolvimento da equipe de saúde no aconselhamento e acompanhamento da mulher que amamenta e daquela que deseja iniciar a alimentação complementar.

A prática da alimentação complementar pode ter ligação com fatores culturais e regionais, necessitando de um estudo mais aprofundado, de modo a identificar a interferência sociocultural no estabelecimento da alimentação complementar e do AM.

\section{Referências}

1. Brasil. Ministério da Saúde. Saúde da criança: nutrição infantil: aleitamento materno e alimentação complementar. Brasília: Ministério da Saúde; 2009. Cadernos de Atenção Básica, 23.

2. Frota MA, Costa FL, Soares SD, Filho OAS, Albuquerque CM, Casimiro CF. Fatores que interferem no aleitamento materno. Rev. Rene 2009; 10(3):61-7.

3. Kemp TA. Revolução industrial na Europa do século XIX. Lisboa: Edições 70; 1987.

4. Vinagre RD, Diniz EMA, Vaz FAC. Leite humano: um pouco de história. Rev. Pediatria 2001; 23(4):340-345.

5. Venâncio SI, Monteiro CA. A tendência da prática da amamentação no Brasil nas décadas de 70 e 80. Rev. Bras. Epidemiologia 1998; 1(1):40-49. 
6. Brasil. Sistema de Informação da Atenção Básica. Município de Ribeirão das Neves. [Acesso em 10 ago. 2011]. Disponível em: http://www2.datasus.gov.br/SIAB/index.php?area=0201

7. Brasil. Ministério da Saúde. II Pesquisa de prevalência de aleitamento materno nas capitais brasileiras e Distrito Federal. Brasília: Ministério da Saúde; 2009.

8. Lopes LFD. Apostila estatística. Santa Maria: DE- UFSM; 2003.

9. Arantes CIS, Oliveira MM, Vieira TCR, Beijo LA, Gradim CVC, Goyatá SLT. Aleitamento materno e práticas alimentares de crianças menores de seis meses em Alfenas, Minas Gerais. Rev. Nutr. 2011; 24(3):421-429.

10. Filamingo BO, Lisboa BCF, Basso NAS. A prática do aleitamento materno entre mães adolescentes na cidade de Dois Córregos, estado de São Paulo. Rev. Scientia Medica 2012; 22(2):81-85.

11. Volpini CCA, Moura EC. Determinantes do desmame precoce no distrito noroeste de Campinas. Rev. Nutr. 2005; 18(3):311-19.

12. Brasil. Ministério da Saúde. Dez passos para uma alimentação saudável: guia alimentar para crianças menores de dois anos: um guia para o profissional da saúde na atenção básica. 2. ed. Brasília: Ministério da Saúde; 2010.

13. Caetano MC, Ortiz TTO, Silva SGL, Souza FIS, Sarni ROS. Alimentação complementar: práticas inadequadas em lactentes. J. Pediatria 2010; 86(3):196-201.

14. Vieira GO, Silva LR, Vieira TO, Almeida JAG, Cabral VA. Hábitos alimentares de crianças menores de 1 ano amamentadas e não amamentadas. J. Pediatria 2004; 80(5):411-416.

15. Monte CMG, Giugliani ERJ. Recomendações para alimentação complementar da criança em aleitamento materno. J. Pediatria 2004; 80(Supl. 5):S131-S142.

16. Strassburger SZ, Vitolo MR, Bortolini GA, Pitrez PM, Jones MH, Stein RT. Erro alimentar nos primeiros meses de vida e sua associação com asma e atopia em pré-escolares. J. Pediatria. 2010; 86(5):391-398.

17. Gomes GP, Gubert MB. Aleitamento materno em crianças menores de 2 anos e situação domiciliar quanto à segurança alimentar e nutricional. J. Pediatria 2012; 88(3):279-282. 
